\title{
Optical spectroscopy of galaxies in the direction of the Virgo cluster $^{\star}$
}

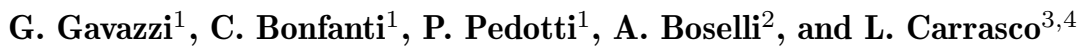 \\ 1 Università degli Studi di Milano - Bicocca, P.zza dell'Ateneo Nuovo 1, 20126 Milano, Italy \\ 2 Laboratoire d'Astronomie Spatiale, Traverse du Siphon, F-13376 Marseille Cedex 12, France \\ 3 Instituto Nacional de Astrofísica, Optica y Electrónica, Apartado Postal 51, CP 72000 Puebla, Pue., Mexico \\ ${ }^{4}$ Observatorio Astronómico Nacional/UNAM, Ensenada B.C., Mexico
}

Received May 23; accepted July 7, 2000

\begin{abstract}
Optical spectroscopy of 76 galaxies, 48 of which are projected in the direction of the Virgo cluster and 28 onto the Coma-A1367 supercluster, is reported. Adding these new measurements to those found in the literature, the redshift completeness in the Virgo region becomes $92 \%$ at $B_{\mathrm{T}} \leq 16.0$ and $68 \%$ at $B_{\mathrm{T}} \leq 18.0$. The one of CGCG galaxies in the direction of the Coma-A1367 supercluster becomes $98 \%$. The Virgo cluster membership estimates obtained on morphological grounds by Binggeli et al. (1985) are confirmed in all cases. However, several "possible members" classified as BCD (if in the cluster) are found instead to be giant emission-line galaxies in the background of the Virgo cluster
\end{abstract}

Key words: galaxies: clusters; virgocluster — galaxies: distances and redshifts

\section{Introduction}

The Virgo cluster, the nearest rich cluster of galaxies in the northern hemisphere, was surveyed with unprecedented resolution and completeness in the extensive photographic survey carryed out with the DuPont telescope at Las Campanas. Based on this material Binggeli et al. (1985) compiled the Virgo Cluster Catalogue (VCC), which lists 2096 galaxies brighter than $B_{\mathrm{T}} \leq 20.0(1323$ of which constitute a complete subsample to $B_{\mathrm{T}} \leq 18.0$ ). This work has served to many important developements,

Send offprint requests to: Gavazzi@uni.mi.astro.it

* Based on observations obtained with the Loiano telescope belonging to the University of Bologna, Italy, with the OHP, operated by the French CNRS and with the G. Haro telescope of the INAOE, Mexico. in particular for mapping for the first time the luminosity function of a cluster down to $M_{\mathrm{p}}=-13.1$ (assuming a distance modulus of Virgo $\mu=-31.1$, corresponding to the distance of $17 \mathrm{Mpc}$; see Gavazzi et al. 1999a) and for comparing the various luminosity functions across the entire Hubble sequence (Sandage et al. 1985). In absence of a complete redshift information (by the time of its publication only about $30 \%$ VCC galaxies had an actual redshift measurement), the cluster membership was assigned to the individual objects on a purely morphological (surface brightness) basis. Since then the number of available redshifts either from optical (e.g. Binggeli et al. 1985; Grogin et al. 1998; hereafter GGH98) or from radio spectroscopy (e.g. Haynes \& Giovanelli 1986; Hoffman et al. 1987; Hoffman et al. 1989; Hoffman et al. 1995; Magri 1994) has increased considerably. Nonetheless the present redshift completeness in this region is still only $65 \%$ at $B_{\mathrm{T}} \leq 18.0$.

A better redshift completeness exists in the ComaA1367 supercluster region $11^{\mathrm{h}} 30^{\mathrm{m}}<\alpha<13^{\mathrm{h}} 30^{\mathrm{m}} ; 18^{\circ}<$ $\delta<32^{\circ}$, limited however to the shallower magnitude limit of 15.7 of the CGCG catalogue (Zwicky et al. 1961-68). Gavazzi et al. (1999b) counted 1068 redshift measurements out of 1127 CGCG galaxies listed in this region. With the aim of contributing with new redshift measurements in these regions we undertook the spectroscopic survey presented in this paper, which was carryed out during marginally photometric nights. The observations and data reduction are presented in Sect. 2. The new redshifts are given and discussed in Sect. 3 .

\section{Observations and data reduction}

Galaxies in the present study were primarily selected among the objects brighter than $B_{\mathrm{T}} \leq 17.0$ in the VCC Catalogue of Virgo Cluster galaxies by Binggeli et al. (1985). CGCG (Zwicky et al. 1961-68) 
Table 1. The spectrograph characteristics

\begin{tabular}{lcccccc}
\hline Telescope & run & Spectrograph & $\begin{array}{c}\text { dispersion } \\
\AA / \mathrm{mm}\end{array}$ & $\begin{array}{c}\text { coverage } \\
\AA\end{array}$ & CCD type & $\begin{array}{c}\text { pix } \\
\mu \mathrm{m}\end{array}$ \\
\hline Loiano & Jan.-Feb. 1999 & BFOSC & 198 & $4060-7900$ & $1024 \times 1024 \mathrm{TH}$ & 19 \\
Loiano & Jan.-Feb. 2000 & BFOSC & 198 & $3600-8900$ & $1340 \times 1300 \mathrm{EEV}$ & 20 \\
Cananea & Apr. 2000 & LFOSC & 228 & $4000-7100$ & $576 \times 384 \mathrm{TH}$ & 23 \\
OHP & Mar. 1999 & CARELEC & 133 & $3200-7100$ & $2048 \times 1024 \mathrm{EEV}$ & 13.5 \\
\hline
\end{tabular}
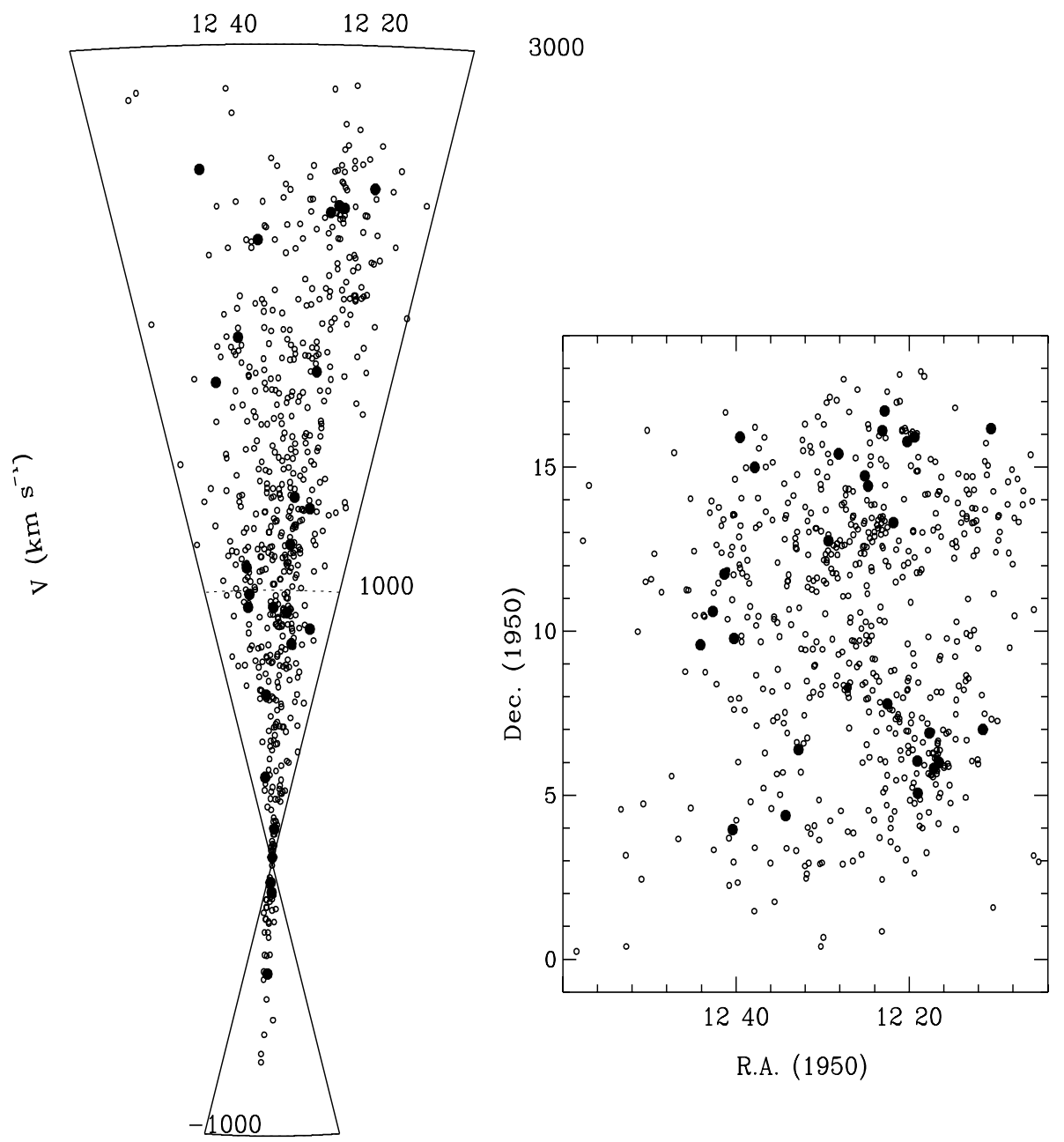

Fig. 1. The distribution in celestial coordinates of 639 Virgo galaxies with $V \leq 3000 \mathrm{~km} \mathrm{~s}^{-1}$ (right) and a wedge diagram (left). The filled symbols represent measurements obtained in the present work

galaxies in the region $11^{\mathrm{h}} 30^{\mathrm{m}}<\alpha<13^{\mathrm{h}} 30^{\mathrm{m}} ; 18^{\circ}<$ $\delta<32^{\circ}$, containing the Coma-A1367 supercluster, were also selected as filler objects. Long-slit, low dispersion spectra of 76 galaxies were obtained in several observing runs since 1999 using the imaging spectrographs BFOSC and LFOSC attached to the Cassini $1.5 \mathrm{~m}$ telescope at Loiano (Italy), to the $2.1 \mathrm{~m}$ telescope of the Guillermo Haro Observatory at Cananea (Mexico), respectively, and with the CARELEC spectrograph (Lemaitre et al. 1990) attached to the $1.92 \mathrm{~m}$ telescope of the Observatoire de Haute Provence (OHP) (France).
Table 1 lists the characteristics of the instrumentation in the adopted set-up.

The observations at Loiano were performed using a 2.0 or 2.5 arcsec slit, depending on the seeing conditions, generally oriented E-W. Every galaxy spectrum was preceded and followed by an exposure of a HeAr lamp to secure the wavelength calibration. The exposure time ranged between 20 and 90 min (1999 run) according to the brightness of the target objects, or 15 min (2000 run) owed to the much higher quantum efficiency of the new EEV detector. 
1240

1220
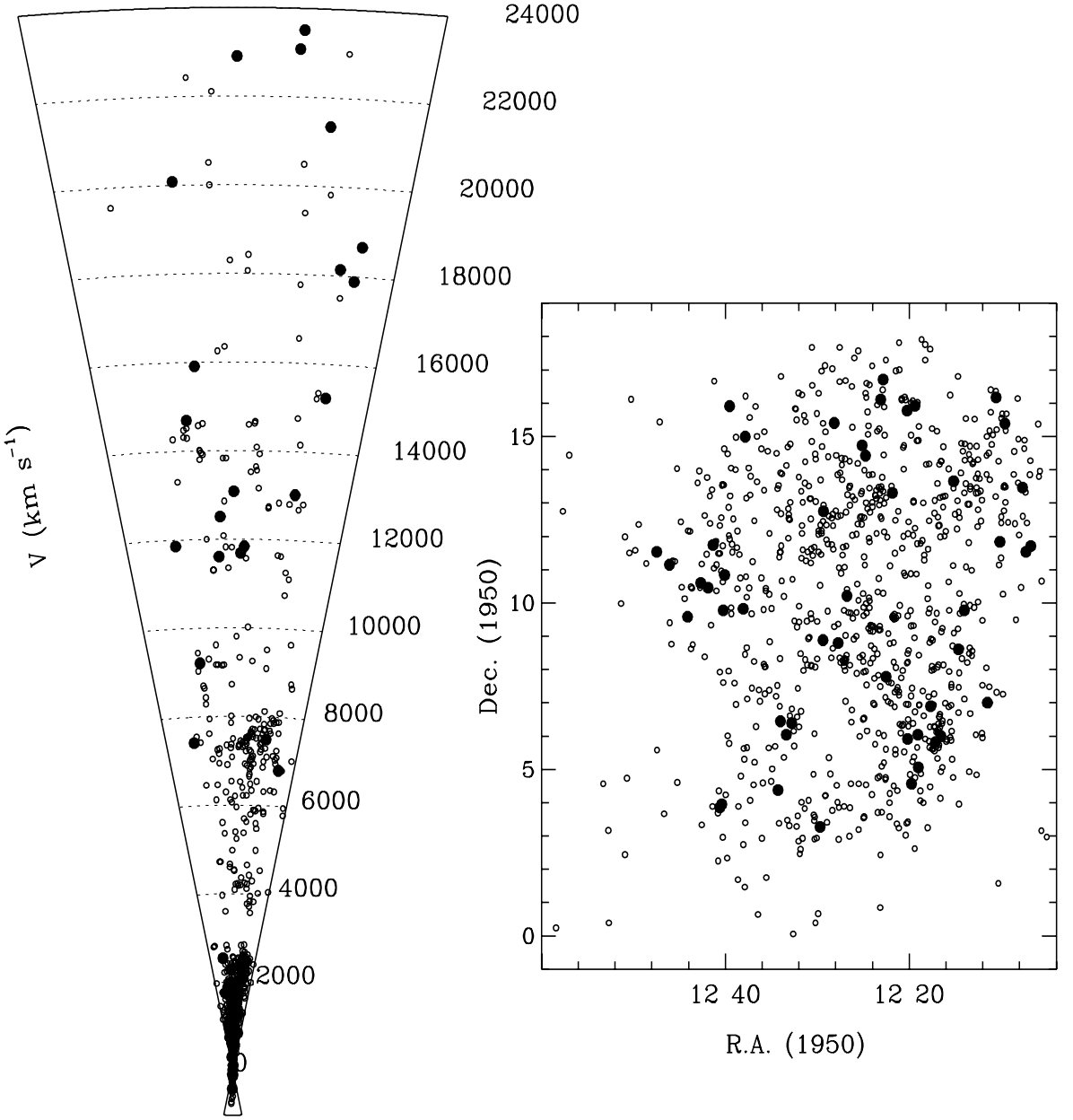

Fig. 2. The distribution in celestial coordinates of 913 Virgo galaxies with $V \leq 24000 \mathrm{~km} \mathrm{~s}^{-1}$ (right) and a wedge diagram (left) (same symbols as in Fig. 1)

The observations at Cananea were carried out with a 1.9 arcsec slit, generally oriented N-S. Every galaxy spectrum was preceded and followed by an exposure of a XeNe lamp to secure the wavelength calibration. The exposure time ranged between 20 and 40 min according to the brightness of the target objects.

The observations at OHP were carried out with a 2.5 arcsec fixed slit, generally oriented E-W. Every galaxy spectrum was preceded and followed by an exposure of a HeAr lamp to secure the wavelength calibration. The exposure time ranged between 20 and 30 min according to the brightness of the target objects. In all runs the observations were obtained in nearly photometric conditions, with thin cirrus. The orientation of the slit was modified from the set-up given above when two adiacent objects were observable in the same exposure.

The data reduction was performed in the IRAF-PROS environment. After bias subtraction, when 3 or more frames of the same target were obtained, these were combined (after spatial alignment) using a median filter to help cosmic rays removal. Otherwise the cosmic rays were removed under visual inspection. The wavelength calibration was checked on known sky lines. These were found within $\sim 1 \AA$ from their nominal value, providing an estimate of the systematic uncertainty on the derived velocities of $\sim 50 \mathrm{~km} \mathrm{~s}^{-1}$. After subtraction of the sky background, one-dimensional spectra were extracted from the frames. These spectra were analyzed with either of two methods:

1) individual line measurement: all spectra taken at Loiano 2000 were inspected and emission/absorption lines were identified. Emission lines include $\mathrm{H} \alpha$, [NII] and [SII]. Absorption lines include the $\mathrm{MgI}, \mathrm{Ca}-\mathrm{Fe}$ and $\mathrm{Na}$. The galaxy redshift was obtained from these individual measurements. If more than one line was identified, the galaxy redshift was derived as the weighted mean of the individual measurements, with weights proportional to the line intensities;

2) cross correlation technique: spectra obtained in all the remaining runs were analyzed using the cross-correlation technique of Tonry \& Davis (1979). This method is based on a "comparison" between the spectrum 


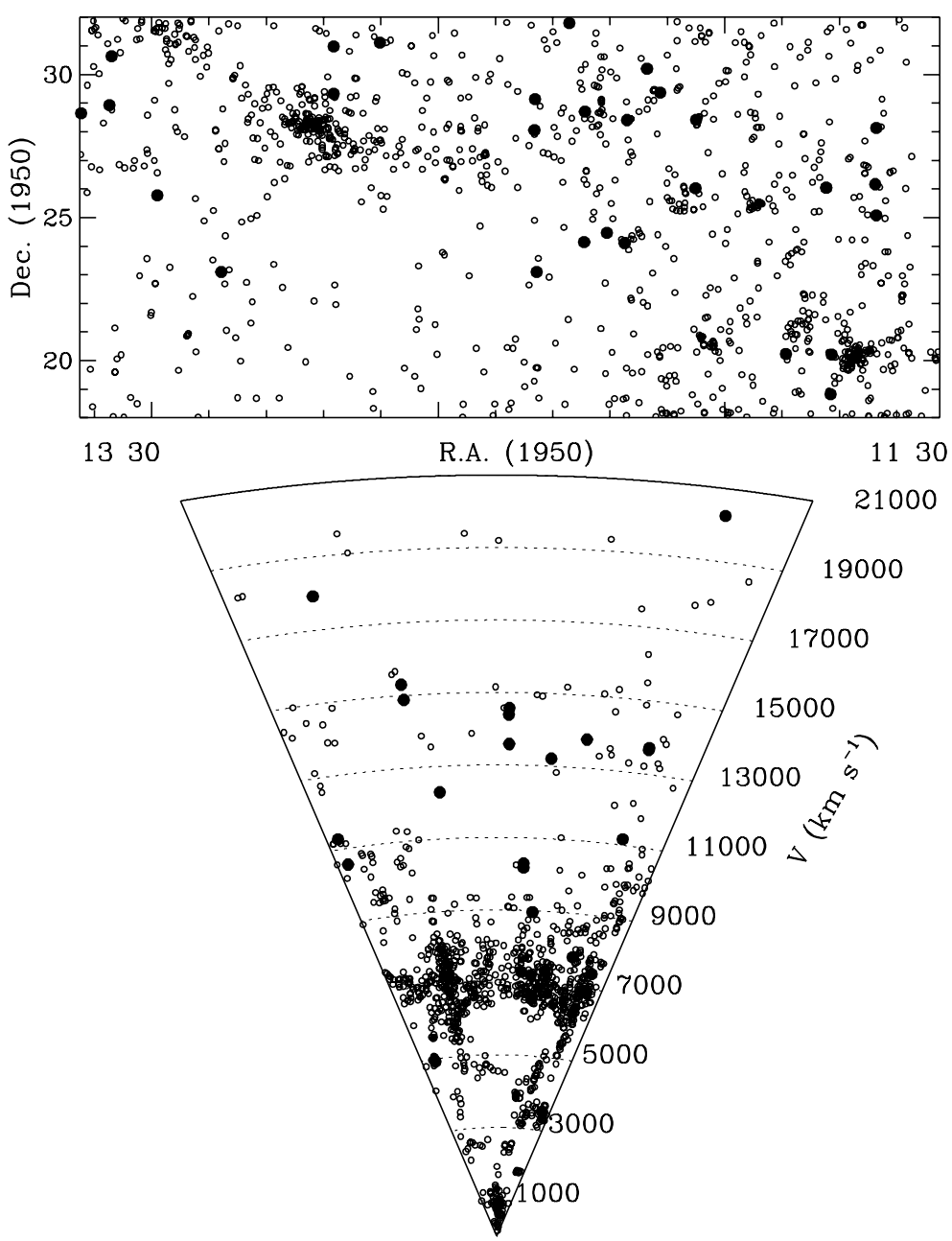

Fig. 3. The distribution in celestial coordinates of 1109 galaxies in the direction of the Coma supercluster with measured redshift (top) and a wedge diagram (bottom) (same symbols as in Fig. 1)

of a galaxy whose redshift is to be determined, and a fiducial spectral template of a galaxy (or star) of appropriate spectral type to contain the wanted absorption/emission lines. The basic assumption behind this method is that the spectrum of a galaxy is well approximated by the spectrum of its stars, modified by the effects of the stellar motions inside the galaxy and by the systemic redshift. For this purpose high signal-to-noise spectra were taken of four template galaxies: M 105 and M 32 (absorption lines) and VCC 1554 and IC 342 (emission lines), which were converted to the restframe $\lambda$. The observed redshifts $\left(V_{\text {obs }}\right)$ were not transformed to Heliocentric.

\section{Results}

The velocity measurements obtained in this work are listed in Table 2 (Virgo) and 3 (Coma) as follow:

Column 1: the CGCG (Zwicky et al. 1961-68) or VCC (Binggeli et al. 1985) designation.

Columns 2, 3: (B1950) celestial coordinates, measured with few arcsec uncertainty.
Column 4: morphological type as given in the VCC.

Column 5: photographic magnitude.

Columns 6, 7: observed recessional velocity with uncertainty derived in this work. The latter quantity includes only statistical errors. The global uncertainty can be derived by adding in quadrature the systematic error of $50 \mathrm{~km} \mathrm{~s}^{-1}$ due to the uncertainty in the absolute wavelength calibration. Several absorption line spectra have statistical errors up to $900 \mathrm{~km} \mathrm{~s}^{-1}$, reflecting a lack of strong features. These redshifts, however, are sufficient to derive a membership.

Column 8: type of lines ( $\mathrm{A}=$ absorption; $\mathrm{E}=$ emission). Column 9: observing run (L99 $=$ Loiano 1999, L00 = Loiano 2000, Can = Cananea 2000, OHP = OHP 1999). Column 10: old membership as given in the VCC (bk = background, $\mathrm{m}=$ member, $-=$ possible member) (only for Table 2).

Column 10a: new membership.

Columns 11, 12: previously available redshift, with reference. 
Table 2. Parameters of the observed Virgo galaxies

\begin{tabular}{|c|c|c|c|c|c|c|c|c|c|c|c|c|}
\hline $\begin{array}{l}\text { Gal. } \\
\text { (1) }\end{array}$ & $\begin{array}{c}\mathrm{RA}(1950) \\
\mathrm{h} \mathrm{m} \mathrm{s} \\
(2)\end{array}$ & $\begin{array}{c}\text { Dec. }(1950) \\
\text { o }, 11 \\
(3)\end{array}$ & $\begin{array}{c}\text { Type } \\
(4)\end{array}$ & $\begin{array}{c}B_{\mathrm{T}} \\
\operatorname{mag}_{(5)}\end{array}$ & $\begin{array}{c}V \\
\mathrm{~km} \mathrm{~s}^{-1} \\
(6)\end{array}$ & $\begin{array}{l} \pm \\
(7)\end{array}$ & $\begin{array}{c}\text { Lines } \\
(8)\end{array}$ & $\begin{array}{l}\text { Run } \\
(9)\end{array}$ & $\begin{array}{c}\text { Memb } \\
(10)\end{array}$ & $\begin{array}{c}\text { new Memb } \\
\text { (10a) }\end{array}$ & $\begin{array}{l}v_{\text {alt }} \\
(11)\end{array}$ & $\begin{array}{l}\text { ref } \\
(12)\end{array}$ \\
\hline VCC 0007 & 120645.60 & 114230.0 & $\mathrm{Sc}$ & 15.04 & 18675 & 425 & A & Can & bk & bk & & \\
\hline VCC 0014 & 120717.80 & 113205.0 & $\mathrm{BCD} ?$ & 16.50 & 17891 & 35 & $\mathrm{E}$ & L00 & - & $\mathrm{bk}$ & & \\
\hline VCC 0019 & 120740.80 & 132800.0 & BCD? & 16.50 & 6803 & 99 & $\mathrm{E}$ & L00 & - & bk & & \\
\hline VCC 0045 & 120934.70 & 152315.0 & BCD? & 16.00 & 15236 & 36 & $\mathrm{E}$ & L00 & - & bk & & \\
\hline VCC 0064 & 121008.50 & 114955.0 & $\mathrm{Sab}$ & 15.04 & 18142 & 336 & A & Can & bk & bk & & \\
\hline VCC 0074 & 121031.80 & 161024.0 & BCD? & 16.30 & 861 & 78 & $\mathrm{E}$ & Can & - & $\mathrm{m}$ & & \\
\hline VCC 0099 & 121128.90 & 70004.0 & $\mathrm{Sa} ?$ & 14.81 & 2476 & 214 & $\mathrm{E}$ & L99 & - & $\mathrm{m}$ & 2444 & GGH98 \\
\hline VCC 0196 & 121400.00 & 94624.0 & $\mathrm{BCD} ?$ & 16.50 & 13024 & 57 & $\mathrm{E}$ & L00 & - & bk & & \\
\hline VCC 0225 & 121439.00 & 83612.0 & $\mathrm{BCD} ?$ & 17.00 & 21345 & 126 & $\mathrm{E}$ & L00 & - & bk & & \\
\hline VCC 0249 & 121509.00 & 133930.0 & $\mathrm{Sa}$ & 14.61 & 7491 & 61 & $\mathrm{E}$ & Can & bk & $\mathrm{bk}$ & & \\
\hline VCC 0323 & 121633.20 & 60012.0 & $\mathrm{Sa}$ & 14.91 & 2402 & 358 & A & L99 & - & $\mathrm{m}$ & 2756 & GGH98 \\
\hline VCC 0362 & 121709.00 & 54856.0 & $\mathrm{Sa}$ & 14.51 & 1300 & 304 & A & L99 & - & $\mathrm{m}$ & 1536 & GGH98 \\
\hline VCC 0397 & 121739.00 & 65402.0 & $\mathrm{dE} ?$ & 15.00 & 2411 & 809 & A & L99 & - & $\mathrm{m}$ & 2495 & GGH98 \\
\hline VCC 0482 & 121900.80 & 50324.0 & SOa & 14.77 & 1802 & 709 & A & L99 & - & $\mathrm{m}$ & 2170 & GGH98 \\
\hline VCC 0486 & 121903.80 & 60235.0 & S0a & 14.50 & 2386 & 252 & A & L99 & - & $\mathrm{m}$ & 2498 & GGH98 \\
\hline VCC 0510 & 121922.80 & 155518.0 & $\mathrm{dE}$ & 15.13 & 804 & 151 & A & Can & $\mathrm{m}$ & $\mathrm{m}$ & & \\
\hline VCC 0541 & 121945.00 & 43348.0 & $\mathrm{BCD}$ & 16.00 & 23511 & 50 & $\mathrm{E}$ & L00 & - & $\mathrm{bk}$ & & \\
\hline VCC 0573 & 122009.60 & 55454.0 & $\mathrm{Sc}$ & 15.20 & 23083 & 189 & $\mathrm{E}$ & Can & bk & bk & 23083 & NED \\
\hline VCC 0583 & 122014.40 & 154636.0 & $\operatorname{Im}$ & 15.76 & -72 & 475 & A & L99 & $\mathrm{m}$ & $\mathrm{m}$ & & \\
\hline VCC 0723 & 122149.80 & 131824.0 & dS0? & 15.04 & 125 & 50 & A & L00 & - & $\mathrm{m}$ & & \\
\hline VCC 0762 & 122230.00 & 74660.0 & $\mathrm{dE}$ & 15.30 & 1341 & 211 & A & Ohp & $\mathrm{m}$ & $\mathrm{m}$ & & \\
\hline VCC 0794 & 122250.40 & 164224.0 & dS0 & 15.50 & 918 & 817 & A & Ohp & $\mathrm{m}$ & $\mathrm{m}$ & & \\
\hline VCC 0817 & 122306.00 & 160642.0 & $\mathrm{dE}$ & 15.00 & 1168 & 153 & A & Can & $\mathrm{m}$ & $\mathrm{m}$ & & \\
\hline VCC 0991 & 122445.90 & 142525.0 & $\mathrm{dE}$ & 14.70 & -406 & 239 & A & L99 & $\mathrm{m}$ & $\mathrm{m}$ & & \\
\hline VCC 1028 & 122506.60 & 144360.0 & dS0? & 15.70 & 21 & 158 & A & Ohp & - & $\mathrm{m}$ & & \\
\hline VCC 1174 & 122645.80 & 101246.0 & $\mathrm{BCD} ?$ & 15.50 & 11840 & 52 & $\mathrm{E}$ & L99 & - & bk & & \\
\hline VCC 1270 & 122743.80 & 84800.0 & $\mathrm{Sa}$ & 15.00 & 11687 & 440 & A & Can & bk & bk & & \\
\hline VCC 1304 & 122809.00 & 152412.0 & dS0 & 15.50 & -108 & 294 & A & L99 & $\mathrm{m}$ & $\mathrm{m}$ & & \\
\hline VCC 1389 & 122919.80 & 124530.0 & $\mathrm{dE}$ & 15.91 & 936 & 193 & A & Can & $\mathrm{m}$ & $\mathrm{m}$ & & \\
\hline VCC 1395 & 122923.40 & 85248.0 & $\mathrm{dE} ?$ & 16.20 & 22900 & 100 & $\mathrm{E}$ & L00 & - & bk & & \\
\hline VCC 1423 & 122942.60 & 31630.0 & $\mathrm{BCD} ?$ & 16.00 & 13079 & 98 & A & Can & - & $\mathrm{bk}$ & & \\
\hline VCC 1608 & 123247.60 & 62325.0 & $\mathrm{E}$ & 14.20 & 2285 & 193 & A & L99 & - & $\mathrm{m}$ & 2464 & GGH98 \\
\hline VCC 1643 & 123321.00 & 60212.0 & S0 & 15.20 & 12509 & 258 & A & Ohp & - & bk & 12563 & GGH98 \\
\hline VCC 1671 & 123359.60 & 62641.0 & dS0 & 14.80 & 11608 & 809 & $\mathrm{~A}$ & L99 & - & bk & 11846 & GGH98 \\
\hline VCC 1687 & 123416.20 & 42242.0 & $\mathrm{dE}$ & 15.10 & 616 & 122 & A & Can & - & $\mathrm{m}$ & & \\
\hline VCC 1836 & 123749.80 & 145930.0 & dS0 & 14.54 & 1927 & 148 & A & Can & $\mathrm{m}$ & $\mathrm{m}$ & & \\
\hline VCC 1849 & 123803.60 & 94942.0 & BCD? & 16.20 & 15905 & 50 & $\mathrm{E}$ & L00 & - & bk & & \\
\hline VCC 1906 & 123932.20 & 155438.0 & S0 & 15.70 & 314 & 138 & A & Can & - & $\mathrm{m}$ & & \\
\hline VCC 1927 & 124005.40 & 105024.0 & $\mathrm{Sc}$ & 14.91 & 20085 & 180 & A & Can & bk & $\mathrm{bk}$ & & \\
\hline VCC 1936 & 124014.40 & 94654.0 & dS0 & 15.68 & 985 & 276 & A & Ohp & $\mathrm{m}$ & $\mathrm{m}$ & & \\
\hline VCC 1947 & 124023.30 & 35701.0 & $\mathrm{dE}$ & 14.56 & 1083 & 405 & A & L99 & - & $\mathrm{m}$ & 944 & GGH98 \\
\hline VCC 1956 & 124036.00 & 35118.0 & S.. & 15.10 & 14691 & 51 & $\mathrm{E}$ & L99 & - & bk & 14659 & GGH98 \\
\hline VCC 1982 & 124119.20 & 114412.0 & $\mathrm{dE}$ & 15.30 & 938 & 464 & $\mathrm{~A}$ & Ohp & $\mathrm{m}$ & $\mathrm{m}$ & & \\
\hline VCC 1997 & 124151.60 & 102742.0 & $\mathrm{Sb}$ & 15.10 & 9210 & 46 & $\mathrm{E}$ & Can & bk & bk & & \\
\hline VCC 2015 & 124240.20 & 103554.0 & BCD? & 16.20 & 2545 & 115 & $\mathrm{E}$ & L00 & - & $\mathrm{m}$ & & \\
\hline VCC 2042 & 124407.20 & 93448.0 & $\mathrm{dE}$ & 14.84 & 1765 & 154 & A & Can & $\mathrm{m}$ & $\mathrm{m}$ & & \\
\hline VCC 2077 & 124604.50 & 110851.0 & Sab & 15.20 & 11860 & 225 & A & Can & bk & bk & & \\
\hline VCC 2082 & 124727.60 & 113206.0 & S.. & 15.30 & 7421 & 26 & $\mathrm{E}$ & Can & bk & $\mathrm{bk}$ & & \\
\hline
\end{tabular}

Figure 1 gives a representation in celestial coordinates of 639 galaxies in the $\mathrm{VCC}$ region with redshift $V \leq 3000 \mathrm{~km} \mathrm{~s}^{-1}$ (panel a) and a wedge diagram (in the same velocity window) is given in panel b. Small symbols mark galaxies taken from the literature, filled circles mark the measurements obtained in this work. Figure 2 gives a representation in celestial coordinates of 913 galaxies in the $\mathrm{VCC}$ region with redshift $\leq 24000 \mathrm{~km} \mathrm{~s}^{-1}$ (panel a) and a wedge diagram is given in panel b. Figure 3 gives a representation in celestial coordinates of 1109 galaxies in the Coma region with measured redshift (top) and a wedge diagram is given (bottom). (Same use of symbols as in Figs. 1 and 2).

\subsection{Redshift completeness}

The VCC catalogue contains 2096 galaxies brighter than $B_{\mathrm{T}}=20.0$. Of these only 913 have a redshift measurement so far. Even at brighter levels the redshift information is far from complete (see Table 4 for details). For example among the 849 galaxies with $B_{\mathrm{T}}<16.0$ there are still 69 with no spectra available. It is not surprising, though, that 
Table 3. Parameters of the observed Coma galaxies

\begin{tabular}{|c|c|c|c|c|c|c|c|c|c|c|c|}
\hline $\begin{array}{l}\text { Gal. } \\
(1)\end{array}$ & $\begin{array}{c}\mathrm{RA}(1950) \\
\mathrm{h} \mathrm{m} \mathrm{s} \\
(2)\end{array}$ & $\begin{array}{c}\text { Dec. }(1950) \\
\text { o } / \text { " } \\
(3)\end{array}$ & $\begin{array}{c}\text { Type } \\
(4)\end{array}$ & $\begin{array}{c}B_{\mathrm{T}} \\
\mathrm{mag} \\
(5)\end{array}$ & $\begin{array}{c}V \\
\mathrm{~km} \mathrm{~s}_{(6)}^{-1}\end{array}$ & $\begin{array}{l} \pm \\
(7)\end{array}$ & $\begin{array}{c}\text { Lines } \\
(8)\end{array}$ & $\begin{array}{l}\text { Run } \\
(9)\end{array}$ & $\begin{array}{l}\text { Memb } \\
(10 \mathrm{a})\end{array}$ & $\begin{array}{l}v_{\text {alt }} \\
(11)\end{array}$ & $\begin{array}{l}\text { ref } \\
(12)\end{array}$ \\
\hline $127-028$ & 113851.25 & 250457.3 & S0 & 15.60 & 3518 & 330 & A & L99 & $\mathrm{fg}$ & & \\
\hline $127-029 \mathrm{~N}$ & 113859.62 & 260956.6 & $\mathrm{E}$ & 16.30 & 7407 & 14 & $\mathrm{E}$ & Can & $\mathrm{m}$ & & \\
\hline $127-029 \mathrm{~S}$ & 113900.06 & 260926.5 & $\mathrm{E}$ & 16.30 & 6927 & 113 & A & Can & $\mathrm{m}$ & & \\
\hline $97-153 \mathrm{~W}$ & 114513.24 & 184955.0 & S.. & 16.30 & 11156 & 20 & $\mathrm{E}$ & Can & bk & & \\
\hline $97-153 \mathrm{E}$ & 114515.08 & 184936.7 & S.. & 16.30 & 20261 & 112 & $\mathrm{E}$ & Can & bk & & \\
\hline $127-057 \mathrm{~S}$ & 114550.62 & 260223.9 & S.. & 16.50 & 13666 & 75 & $\mathrm{E}$ & Can & bk & & \\
\hline $127-057 \mathrm{~N}$ & 114551.69 & 260251.3 & S.. & 16.50 & 13718 & 15 & $\mathrm{E}$ & Can & bk & & \\
\hline $127-102$ & 115130.07 & 232813.1 & $\mathrm{E}$ & 15.70 & 7799 & 139 & A & Ohp & $\mathrm{m}$ & & \\
\hline $128-028 \mathrm{~W}$ & 120405.12 & 260142.4 & $\mathrm{E}$ & 16.40 & 7353 & 198 & A & Can & $\mathrm{m}$ & & \\
\hline $128-028 \mathrm{E}$ & 120406.94 & 260150.2 & $\mathrm{E}$ & 16.40 & 13788 & 143 & A & Can & bk & & \\
\hline $98-088$ & 120903.25 & 201024.8 & S0 & 15.70 & 6564 & 215 & $\mathrm{~A}$ & Ohp & $\mathrm{m}$ & & \\
\hline $128-055$ & 121122.87 & 220200.8 & S0 & 15.70 & 7227 & 330 & A & L99 & $\mathrm{m}$ & & \\
\hline $98-120$ & 121341.37 & 194405.9 & $\mathrm{E}$ & 15.70 & 13208 & 202 & $\mathrm{~A}$ & Ohp & bk & & \\
\hline $98-127$ & 121409.85 & 183918.2 & $\mathrm{E}$ & 15.70 & 8954 & 203 & $\mathrm{~A}$ & Ohp & bk & & \\
\hline 99-013 & 121638.07 & 193306.1 & $\mathrm{Sc}$ & 15.70 & 7297 & 16 & $\mathrm{E}$ & Ohp & $\mathrm{m}$ & & \\
\hline $128-083$ & 121936.55 & 240846.0 & $\mathrm{E}$ & 15.70 & 10182 & 129 & $\mathrm{E}$ & Ohp & bk & & \\
\hline $128-083 \mathrm{E}$ & 121939.64 & 240847.5 & $\mathrm{E}$ & 17.00 & 10292 & 164 & $\mathrm{~A}$ & Ohp & bk & & \\
\hline $128-085$ & 122150.98 & 212612.2 & $\mathrm{Sc}$ & 15.60 & 914 & 21 & $\mathrm{E}$ & Ohp & $\mathrm{fg}$ & & \\
\hline 99-066 & 122620.69 & 194525.9 & $\mathrm{Sb}$ & 15.70 & 13582 & 86 & $\mathrm{E}$ & Ohp & $\mathrm{bk}$ & & \\
\hline $129-003$ & 122631.44 & 245429.9 & $\mathrm{Sc}$ & 15.70 & 14572 & 9 & $\mathrm{E}$ & Ohp & bk & & \\
\hline $99-067$ & 122635.25 & 191652.4 & $\mathrm{E}$ & 15.70 & 14393 & 250 & $\mathrm{~A}$ & Ohp & bk & & \\
\hline $159-087 \mathrm{E}$ & 124810.87 & 274149.8 & $\mathrm{Sbc}$ & 15.70 & 12286 & 16 & $\mathrm{E}$ & Ohp & bk & & \\
\hline $160-036 \mathrm{~S}$ & 125435.00 & 305820.9 & S0 & 16.00 & 15302 & 177 & $\mathrm{~A}$ & Can & bk & & \\
\hline $160-036 \mathrm{E}$ & 125438.62 & 305831.5 & $\mathrm{E}$ & 16.50 & 14880 & 185 & $\mathrm{~A}$ & Can & bk & & \\
\hline $160-163 \mathrm{~S}$ & 131035.69 & 272401.1 & $\mathrm{E}$ & 16.50 & 17929 & 178 & $\mathrm{~A}$ & Can & bk & & \\
\hline $160-163$ & 131036.56 & 272421.7 & S0a & 15.70 & 18015 & 72 & $\mathrm{E}$ & Can & bk & & \\
\hline $161-029$ & 131912.70 & 263359.0 & $\mathrm{Sb}$ & 15.70 & 4930 & 11 & $\mathrm{E}$ & Ohp & $\mathrm{m}$ & & \\
\hline 161-061S & 132554.00 & 285542.1 & S.. & 15.60 & 11281 & 32 & $\mathrm{E}$ & Can & bk & 11247 & G99 \\
\hline $161-061 \mathrm{~N}$ & 132554.69 & 285657.4 & $\mathrm{E}$ & 16.50 & 10564 & 165 & $\mathrm{~A}$ & Can & bk & & \\
\hline
\end{tabular}

such relatively bright objects remain unmeasured because, given the vicinity of the Virgo cluster, galaxies in this magnitude range have low luminosities $\left(M_{\mathrm{p}}>-15.0\right)$. Given the known inverse proportionality between the luminosity and the surface brightness, they all correspond with extremely low surface brightness galaxies, which are much more difficult to observe spectroscopically than objects of similar magnitude which are further away. Furthermore, galaxies with missing spectra are almost entirely dEs, thus with featureless spectra. During the spectroscopical runs described in the present paper we have tried, unsuccesfully, to measure several of these objects: VCC 236 (dE), 452 (dE), 816 (dE), 1417 (dE), 1497 (dE), $1503(\mathrm{dE}), 1571$ (dE), 1649 (dE), 1755 (dE), 1825 (dIm/dE), 1945 (dE), 1991 (dE), 2083 (dS0) with integration time $\sim 30 \mathrm{~min}$. We point out to those observers who wish to obtain succesfull spectra at $2 \mathrm{~m}$ class telescopes to try with much longer exposures.

The redshift completeness is far better in the Comaregion, limited however to a brighter magnitude. Of the 1127 CGCG galaxies listed in the Coma region, 1082 have $B_{\mathrm{T}} \leq 15.7$. Another 45 belong to multiple systems
Table 4. Redshift completeness in the Virgo cluster

\begin{tabular}{cccc}
\hline mag & tot & with $z$ & $\%$ \\
\hline$\leq 15.0$ & 549 & 546 & 99 \\
$\leq 16.0$ & 849 & 780 & 92 \\
$\leq 17.0$ & 1064 & 864 & 81 \\
$\leq 18.0$ & 1323 & 903 & 68 \\
$\leq 19.0$ & 1704 & 913 & 53 \\
$\leq 20.0$ & 2096 & 916 & 43 \\
\hline
\end{tabular}

which were split in their individual components, each of them fainter than the catalogue limiting magnitude 15.7. Only $2 / 1082$ galaxies with $B_{\mathrm{T}} \leq 15.7$ and $18 / 1127$ with $B_{\mathrm{T}} \leq 16.5$ remain with unknown redshift, thus the sample is $98 \%$ complete.

An interesting example of a strong emission-line object in the background of the Coma supercluster is CGCG 127-057N which was observed at Cananea. The spectrum of this galaxy (see Fig. 4) shows strong Balmer and [OIII] lines and weak [NII] and [SII]. The corresponding 


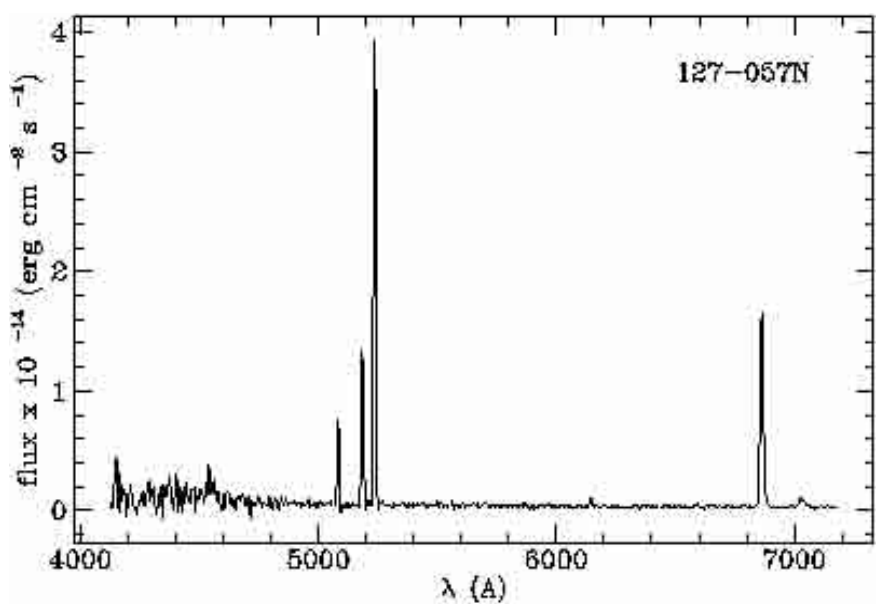

Fig. 4. The emission-line spectrum of 127-057N

metallicity derived from $[\mathrm{OIII}] / \mathrm{H} \beta$, as prescribed by Edmunds \& Pagel (1984) is: $12+\log (\mathrm{O} / \mathrm{H})=8.16$.

\section{2. membership}

The membership to the Virgo cluster given in the VCC (members, possible-members, background) was estimatied on purely morphological grounds (mostly on the surface brightness) by Binggeli et al. (1985). The new redshifts presented in this paper, in conjunction with two other recent sets of Virgo velocity measurements (Magri 1994 and GGH98), can be used to reassess this issue. We find that all objects listed in the VCC as "members" are confirmed as such $\left(V<3000 \mathrm{~km} \mathrm{~s}^{-1}\right)$, stressing the high success-rate of the morphological estimate. "Possible members" are found with $V<3000 \mathrm{~km} \mathrm{~s}^{-1}$ in $67 \%$ of cases, the remaining being background objects. We have noticed that out of the 13 "Possible members" which are in fact high redshift objects, 10 were classified as possible BCDs (BCD?). These appear to be systematically emission-line blue giant objects, not dwarfs, nor compact galaxies (see Table 2).

As an example we give in Fig. 5 the rotation curve of one of them: VCC 1849, which has a total rotational velocity up to $300 \mathrm{~km} \mathrm{~s}^{-1}$, not typical of a dwarf galaxy. The rotation curve was derived at intermediate dispersion during the Loiano 2000 run with the slit oriented along the galaxy major axis. All objects classified as "Background" in the VCC are confirmed as such.

In summary, we obtained 76 new redshift measurements of galaxies, 48 of which are projected onto the Virgo cluster and 28 in the direction of the Coma-A1367 supercluster. With these new data, the redshift completeness in the VCC region remains $92 \%$ for $B_{\mathrm{T}} \leq 16.0$ and $68 \%$ for $B_{\mathrm{T}} \leq 18.0$. All membership estimates, as given in the VCC, are confirmed. We remark that a large fraction of the possible members classified as BCDs?, are found to be giant emission-line galaxies well beyond the Virgo cluster.

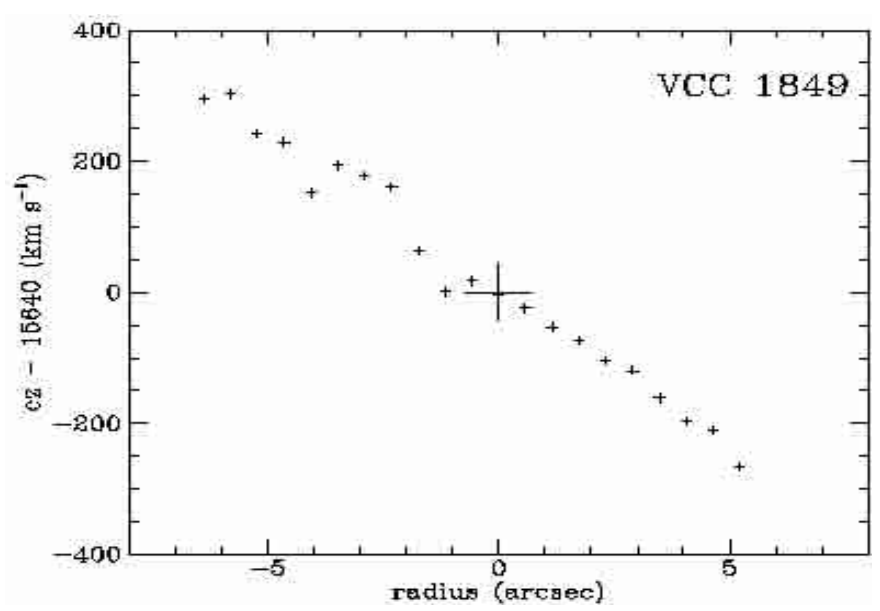

Fig. 5. The $\mathrm{H} \alpha$ rotation curve of VCC 1849

The redshift completeness of CGCG galaxies in the direction of the Coma-A1367 supercluster is now $98 \%$.

Acknowledgements. We wish to thank the TACS of the Loiano, Cananea and OHP telescopes for the generous amounts of time allocated to this project. G.G. wishes to thank his students for their contribution during the observations and the data reduction. This work could not be completed without the use of the NASA/IPAC Extragalactic Database (NED) which is operated by the Jet Propulsion Laboratory, Caltech under contract with NASA. L.C. has had support from CONACYT (Mexico) research grant No. G-28586E.

\section{References}

Binggeli B., Sandage A., Tammann G., 1985, AJ 90, 1681

Binggeli B., Popescu C., Tammann G., 1993, A\&AS 98, 275

Edmunds M., Pagel B., 1984, MNRAS 211, 507

Haynes M., Giovanelli R., 1986, ApJ 306, 466

Hoffman L., Helou G., Salpeter E., Glosson J., Sandage A., 1987, ApJS 63, 247

Hoffman G., Lewis B., Helou G., Salpeter E., Williams B., 1989, ApJS 69, 65

Hoffman G., Lewis B., Salpeter E., 1995, ApJ 441, 28

Gavazzi G., Boselli A., Scodeggio M., Pierini D., Belsole E., 1999a, MNRAS 304, 595

Gavazzi G., Carrasco L., Galli R., 1999b, A\&AS 136, 227 (G99)

Grogin N., Geller M., Huchra J., 1998, ApJS 119, 277 (GGH98)

Lemaitre G., Kohler D., Lacroix D., Meunier J., Vin A., 1990, A\&A 228,540

Magri C., 1994, ApJ 108, 896

Sandage A., Binggeli B., Tammann G., 1985, AJ 90, 395

Tonry J., Davis M., 1979, AJ 84, 1511

Zwicky F., Herzog E., Karpowicz M., Koval C., Wild P., 1961-1968, Catalogue of Galaxies and Clusters of Galaxies, Pasadena: Caltech (GCGC) 